\title{
ANÁLISIS DEL PROCESO EXPORTADOR DE EMPRESAS DEL SECTOR CONFECCIONES DE SANTANDER A ESTADOS UNIDOS*
}

\author{
Edgar Javier Gómez Parada. \\ Gustavo González Sarmiento. ${ }^{2}$
}

\section{Resumen}

Se examina el proceso exportador de los empresarios de confecciones de Santander a Estados Unidos basado en el modelo de internacionalización gradualista (Escuela de Uppsala). El mercado de Estados Unidos es bastante atractivo para los exportadores de confecciones, por el tamaño de las importaciones ( 83 mil millones de dólares en el 2008) y el crecimiento de las mismas $(29,6 \%$ entre el $2002-2008)$. La amplia participación de competidores y la evidente dominancia de China y otros países asiáticos, hace cada vez más fuerte la competencia por el mercado. Los exportadores santandereanos tienen alta permanencia en el mercado y realizan las operaciones de comercio exterior de manera directa, por lo que se ubican en la fase intermedia del proceso de internacionalización.

\section{Palabras clave}

Exportación de confecciones, proceso exportador, exportaciones santandereanas.

\begin{abstract}
It examines the process of business exporters of apparel from Santander to the United States based on the gradualist model of internationalization (School of Uppsala). The United States market is quite attractive for exporters of garments, because the size of imports ( 83 billion dollars in 2008) and growth of the market (29.6\% between 2002 - 2008). Broad participation of competitors and obvious dominance of China and other Asian countries, makes the competition for the market increasingly strong. Santander exporters have high permanence in the market and carry out foreign trade operations directly, so they are located in the middle phase of the internationalization process.
\end{abstract}

\section{Key words}

Apparel exports, export process, Santander exports.

\footnotetext{
* El presente artículo deriva de la investigación denominada "Dinámica competitiva de los sectores calzado y confecciones de Santander y Colombia en Estados Unidos" Proyecto del grupo ESTRATEGIA, Facultad de Negocios Internacionales. USTA.

1 Magíster en Educación. Docente Investigador Facultad de Negocios Internacionales, Universidad Santo Tomás. Correo E: gusta_gsar80@hotmail.com

2 Especialista en Planeación y Administración del Desarrollo Regional. Docente Investigador Facultad de Negocios Internacionales, Universidad Santo Tomás. Correo E: edjagomezp@yahoo.com
} 


\section{Introducción}

El proceso de internacionalización se fundamenta en la teoría económica asociada a la competitividad tratando de dar explicaciones teóricas del comercio internacional.

La internacionalización puede definirse como el proceso mediante el cual se lleva a cabo negocios internacionales en el que intervienen actores (empresas) de dos o más países. Este concepto planteado por Daniel y Radebaugh (2000) sirve de marco explicativo desde diversas dimensiones: los países crean las condiciones para que las empresas puedan tener ambientes favorables que les permitan tener ventajas sobre sus competidores. Desde allí se hace posible la aparición de ventajas comparativas que posteriormente se traducen en competitivas. Otra dimensión es el tipo de "vínculo" que se establece entre las empresas que realizan los negocios internacionales, Rialph (1999) interpreta la internacionalización como aquel conjunto de operaciones que facilita el establecimiento de vínculos más o menos estables entre la empresa y los mercados internacionales, a lo largo de un proceso de creciente implicación y proyección comercial.

Se hace necesario dar mayor amplitud al enfoque y apoyarse en un concepto que recoja elementos de estabilidad y permanencia en los mercados internacionales, mas cuando se trabaja con empresas PYMES, ya que no se puede considerar la internacionalización un único y esporádico esfuerzo que, a veces, al azar le representa a la empresa una exportación. Para ello se toma el concepto de Rialp (1999), que define la internacionalización como:

"todo aquel conjunto de operaciones que facilitan el establecimiento de vínculos
más o menos estables entre la empresa y los mercados internacionales, a lo largo
de un proceso de creciente implicación y proyección internacional de la misma;
esto es, un proceso amplio y complejo de definición de compromiso internacional
adoptado por la organización. Por tanto, se contempla toda una amplia gama o
variedad de fórmulas institucionales y/o vías de penetración alternativas en el
mercado exterior, susceptibles de modificarse en el tiempo, a medida que la
empresa va redefiniendo sus niveles relativos de presencia, compromiso de
recursos, control y/o riesgo operativo a escala internacional"

Este es un concepto sobre el cual se puede soportar diversos enfoques del proceso de internacionalización por diversas razones: primero, porque establece criterios del vínculo de las empresas en términos de estabilidad; segundo, porque exige a la empresa mayor implicación en el proceso mismo; tercero, porque asegura a la empresa proyección internacional (derivado del anterior); cuarto, porque la internacionalización debe ser una decisión adoptada por la organización, es decir, no son esfuerzos aislados de vendedores o agentes (y menos de intermediarios) sino una decisión gerencial; quinto, desde la decisión de la organización de internacionalizarse 
debe diseñarse e implementarse las estrategias para que ello ocurra; un elemento adicional es la apuesta de recursos (inversiones) y control para asegurar la efectividad del proceso.

Las empresas toman la decisión de llegar a mercados extranjeros por varias razones, para Daniel y Radebaugh (2000), las principales son el incremento de las ventas, la adquisición de recursos, la diversificación de sus fuentes de ventas y suministros y la reducción del riesgo competitivo. La intención de internacionalizarse proviene, de acuerdo con López y Ruiz (2000), de fuerzas generadas al interior de la empresa o de condiciones y características del entorno.

Dentro de los enfoques teóricos explicativos de la internacionalización que respondan a modelos característicos de las empresas exportadoras de confecciones de Santander (ubicadas como PYMES) se pueden tener en cuenta el modelo de Uppsala, la teoría ecléctica de Dunning y la teoría de redes, entre otras.

En el enfoque de Dunning, la decisión de internacionalización de la empresa conjuga tres elementos: posesión de activos intangibles que le dan a la empresa ventaja sobre empresas de otros países; dichos activos están relacionados con la tecnología, con aspectos de propiedad intelectual, innovación y posicionamiento de marca, entre otros. Un segundo elemento se asocia con ventajas al momento de la internacionalización basada en la reducción de los costos de transacción y minimización de externalidades. El tercer elemento está representado en las ventajas que ofrece el país en donde se ubican las mercancías. El modelo de Dunning indica que cada uno de estos elementos puede variar en cada caso específico.

La teoría de redes argumenta que la internacionalización es un proceso gradual y surge de cómo la empresa logre establecer y consolidar relaciones de tipo empresarial y social que se traduzcan en posicionamiento en el mercado.

Dentro del presente estudio se asume la internacionalización de las empresas como un proceso gradual y evolutivo que facilita la decisión de la empresa para ingresar a mercados externos, modelo que se conoce como enfoque gradualista, derivado de estudios realizados en la Escuela de Uppsala (Johanson y WiedersheimPaul, 1975), citado por Galván (2003). La gradualidad implica una serie de fases asociadas a mayor implicación de la empresa en las variables del proceso a medida que se avanza en las etapas de internacionalización, con la adquisición de conocimiento del mercado y de las operaciones de comercio exterior (Riaph, 1999). Las etapas de la internacionalización, según el modelo son: no exportar, exportar, organización de redes comerciales propias para realización de exportaciones y ubicación de establecimientos y unidades productivas en los mercados extranjeros.

Cuando la empresa decide exportar, comienza a hacerlo a mercados que son psicológicamente cercanos, es decir, a aquellos con los que encuentra similitudes culturales, de idioma, de prácticas productivas y de consumo y aspectos políticos, entre 
otros. El concepto de distancia psicológica está asociado a la facilidad o dificultad del flujo de información entre la empresa y el mercado, (Johanson y WiedersheimPaul, 1990), citado por Olivares M. A. (2005) y tiene en cuenta los aspectos mencionados anteriormente. La primera fase del proceso de internacionalización se caracteriza además porque las exportaciones son esporádicas, hecho que se refleja en la baja permanencia de las empresas en los mercados destino; es decir, que no todas las empresas logran consolidarse en los mercados y continuar el proceso de internacionalización a fases más avanzadas. La empresa, luego de adquirir conocimiento y experiencia tanto del mercado como del proceso exportación, dirige los productos a mercados psicológicamente más lejanos, que pueden ofrecer mayores oportunidades y mejores dividendos.

El modelo gradualista o enfoque Uppsala, puede complementarse además con los modos de entrada de las empresas a los mercados internacionales; principalmente en el proceso de exportación. De acuerdo a este modelo, Alonso y Donoso (1991), las empresas pueden realizar la exportación de manera directa o indirecta. Cuando la empresa hace exportación directa asume y controla todas las operaciones de comercio exterior, tanto de marketing como de logística. Se distingue que la exportación es directa, cuando la empresa exportadora hace el contacto y la venta directamente al comprador extranjero; o cuando dispone de personal de ventas o agentes que buscan los clientes y toman los pedidos en mercados externos; hay que tener en cuenta que la exportación directa requiere contratar servicios de intermediación para los procesos logísticos y aduaneros.

Cuando se hace de manera indirecta, utiliza intermediarios en el proceso de comercio exterior como agentes, comercializadoras, a los cuales entrega mercancía para que la revendan. Dichos comercializadores, son los que se encargan de ingresar los productos al mercado destino pero, a la vez, obtienen las ganancias, porque adquieren el producto al precio del mercado local.

Las empresas exportadoras de confecciones de Santander a Estados Unidos son pocas; según el registro de exportadores de Proexport Colombia ${ }^{3}$; en el 2007 se registraron 39 empresas. La característica principal de dichas empresas es que son microempresas en su gran mayoría y algunas pequeñas empresas. No se registran grandes empresas del sector confecciones en Santander.

El presente estudio asume la exportación como el modelo de internacionalización predominante por las empresas de confecciones de Santander, no se registra ninguna empresa del sector confecciones originaria de la región, que realice operaciones mediante redes comerciales (ejemplo franquicias); o que haya establecido unidades de producción y comercialización en Estados Unidos.

3 Organización gubernamental, adscrita al Ministerio de Comercio, Industria y Turismo, encargada de la promoción comercial de las exportaciones no tradicionales, el turismo internacional y la Inversión Extranjera en Colombia. 
Para las empresas de confecciones de Santander, el mercado norteamericano es psicológicamente lejano, debido a las diferencias culturales y de idioma; esquemas productivos, modelos de comercialización, características de consumo, exigencias del mercado, normas de calidad, presentación y etiquetado de productos y a la distancia geográfica, entre otras.

Dentro del enfoque gradualista en el proceso de internacionalización los mercados psicológicamente distantes son más atractivos. Específicamente, para los empresarios colombianos de confecciones, el mercado norteamericano es atractivo por:

- Actualmente Estados Unidos es el mayor importador del mundo en confecciones. En el año 2008 las importaciones de confecciones de Estados Unidos alcanzaron un valor de US\$ 83.478.000.000 (ochenta y tres mil cuatrocientos setenta y ocho millones de dólares); con crecimiento de $29,6 \%$ entre el $2002-2008^{4}$.

- El tipo de cambio (entre el peso y el dólar) deja altos dividendos a los empresarios exportadores. A pesar de que en el 2008 el peso colombiano experimentó una revaluación frente al dólar, hecho que lleva a que los empresarios exportadores reciban menos pesos al hacer el cambio de moneda.

- Estados Unidos ofrece ventajas arancelarias a la mayoría de productos de confecciones provenientes de Colombia, contemplados dentro del ATPDEA ${ }^{5}$.

\section{Exportaciones de Confecciones de Colombia y Santander}

Las exportaciones de confecciones de Colombia a todo destino en el 2008, alcanzaron un valor de US\$ 1332 millones de dólares, con participación del 3,54\% del total de las exportaciones nacionales; y, experimentaron un crecimiento de $133,4 \%$ entre el año 2002 - 2007. Específicamente a Estados Unidos el sector confecciones de Colombia exportó US\$ 285 millones de dólares en el 2008, con participación del $2 \%$ del total de exportaciones colombianas a Estados Unidos, sin experimentar crecimiento a dicho mercado entre el 2002 - 2008. Mientras que el valor exportado del sector a Estados Unidos se mantuvo constante en el mismo periodo; esto se refleja en la proporción de confecciones exportadas a Estados Unidos, frente a las exportaciones a todo destino, donde se pasó de $49,8 \%$ en el 2002 , a $21,4 \%$ en el

4 Datos consultados en: Trade Stats Express: http://tse.export.gov

5 Ley de preferencias arancelarias andinas y de erradicación de drogas, incluido en la Ley Comercial por el Presidente de Estados Unidos George Bush, el 6 de agosto de 2002, que otorga preferencias arancelarias a gran cantidad de productos provenientes de Bolivia, Perú, Ecuador y Colombia. Dentro de dichas preferencias se incluyen aproximadamente 300 subpartidas arancelarias del sector confecciones. El ATPDEA es una extensión del ATPA. Actualmente se encuentra vigente y se ha venido prolongando por cortos periodos de tiempo. 
2008. Es evidente que el esfuerzo exportador por parte del sector confecciones, se ha concentrado a mercados geográfica y psicológicamente más cercanos, como Ecuador y Venezuela.

Estos datos reflejan que en exportación de confecciones de Colombia no se ha logrado aumentar la participación en el mercado norteamericano. (Ver cuadro 1). Es decir, que no se ha aprovechado el atractivo mercado y las preferencias arancelarias otorgadas por Estados Unidos a productos provenientes de Colombia para el sector confecciones a través del ATPDEA.

Cuadro 1. Situación exportaciones de confecciones de Colombia Valor en miles dólares fob

\begin{tabular}{|c|c|c|c|c|c|c|c|c|}
\hline & 2002 & 2003 & 2004 & 2005 & 2006 & 2007 & 2008 & $\begin{array}{l}\% \text { VAR } 2002 \\
-2008\end{array}$ \\
\hline $\begin{array}{c}\text { TOTAL EXPORTACIONES } \\
\text { DE COLOMBIA }\end{array}$ & 11879668 & 13128524 & 16729663 & 21185799 & 24367250 & 29717770 & 37625882 & 216,7 \\
\hline $\begin{array}{l}\text { CONFECCIONES A TODO } \\
\text { DESTINO }\end{array}$ & 570836 & 686759 & 922603 & 959120 & 1019972 & 1507889 & 1332049 & 133,4 \\
\hline PART. $\%$ & 4,81 & 5,23 & 5,51 & 4,53 & 4,19 & 5,07 & 3,54 & \\
\hline $\begin{array}{c}\text { TOTAL EXPORTACIONES } \\
\text { DE COLOMBIA A } \\
\text { ESTADOS UNIDOS }\end{array}$ & 5163662 & 5779441 & 6611138 & 8479657 & 9650252 & 10373300 & 14052729 & 172,15 \\
\hline $\begin{array}{l}\text { CONFECCIONES A } \\
\text { ESTADOS UNIDOS }\end{array}$ & 284535 & 439797 & 531565 & 484651 & 432693 & 318802 & 285079 & 0,19 \\
\hline PART. \% & 5,51 & 7,61 & 8,04 & 5,72 & 4,48 & 3,07 & 2,03 & \\
\hline $\begin{array}{c}\text { PROPORCIÓN DE } \\
\text { CONFECCIONES } \\
\text { EXPORTADAS A EEUU }\end{array}$ & 49,8 & 64,1 & 57,6 & 50,5 & 42,4 & 21,1 & 21,4 & \\
\hline
\end{tabular}

Fuente: DANE-DIAN. Elaboración Propia

Las exportaciones de confecciones de Santander alcanzaron el valor de US\$ 31 millones de dólares en el 2008, con participación del 2,32\% del total de Colombia en este sector. Específicamente a Estados Unidos, de Santander se exportaron 7,7 millones de dólares en el 2008, con participación del 2,72\% de las exportaciones de confecciones; la dependencia en el mercado de Estados Unidos es del 25\% (2008) .

Como se ve, las exportaciones de confecciones de Santander a Estados Unidos son bajas y aplica, por tanto, lo mismo expresado sobre exportaciones nacionales del

6 Dependencia del mercado establece la relación del total de exportaciones a todo destino sobre las exportaciones a un mercado específico. En este caso indica que la cuarta parte (25\%) de las exportaciones de confecciones de Santander se dirige a Estados Unidos. 
sector; no se aprovecha la dinámica y preferencias otorgadas por el mercado. (Ver Cuadro 2)

Cuadro 2. Situación exportaciones de confecciones de Colombia y Santander Valor en miles dólares fob

\begin{tabular}{ccccccc}
\hline \multicolumn{2}{c}{$\mathbf{2 0 0 7}$} & & \multicolumn{2}{c}{$\mathbf{2 0 0 8}$} \\
\hline & A TODO & A ESTADOS \\
DESTINO & UNIDOS & $\begin{array}{c}\text { DEPENDENCIA } \\
\text { En el mercado de } \\
\text { EEUU } \\
\%\end{array}$ & $\begin{array}{c}\text { A TODO } \\
\text { DESTINO }\end{array}$ & $\begin{array}{c}\text { A } \\
\text { ESTADOS } \\
\text { UNIDOS }\end{array}$ & $\begin{array}{c}\text { DEPENDENCIA } \\
\text { En mercado } \\
\text { De EUU } \\
\%\end{array}$ \\
$\begin{array}{c}\text { Total Nacional } \\
\text { Exportaciones } \\
\text { Confecciones }\end{array}$ & 1507889 & 318802 & 21,1 & 1332049 & 285079 & 21,4 \\
$\begin{array}{c}\text { Exportación } \\
\text { Confecciones } \\
\text { Santander }\end{array}$ & 38488 & 10468 & 27,2 & 31003 & 7756 & $25 \%$ \\
$\begin{array}{c}\text { Participación } \\
\text { De Santander } \\
\text { en el Nacional }\end{array}$ & $2,55 \%$ & $3,28 \%$ & & $2,32 \%$ & $2,72 \%$ & \\
\hline
\end{tabular}

Fuente: DIAN - Siex. (Sistema Estadístico de Comercio Exterior de Colombia). Elaboración propia

\section{El mercado Norteamericano de confecciones}

El mercado de confecciones en Estados Unidos es bastante amplio y ha estado en permanente crecimiento; además el aumento de las importaciones hace más atractivo el mercado. En el 2008 las importaciones de confecciones de Estados Unidos representaron US\$ 83478 millones de dólares, con crecimiento del 29,6\% entre el 2002 - 2008. Ver Cuadro 3.

Cuadro 3. Importaciones confecciones por Estados Unidos

\begin{tabular}{lc}
\hline $\begin{array}{l}\text { Valor importaciones 2008 } \\
\text { Miles US Fob }\end{array}$ & 83478095 \\
\hline Dinámica 2002-2008 & $29,6 \%$ \\
\hline
\end{tabular}

Fuente: TradeStats Express. http://tse.export.gov. Elaboración propia 
La penetración de las importaciones de confecciones, y en general de prendas de vestir derivadas en el mercado norteamericano ha aumentado. Según reportes del Annual Statistical Analysis of the U.S. Apparel \& Footwear Industries (2008) en el 2007 dicho índice de importaciones para ropa alcanzó un valor de 95\%. Ello indica que el 95\% del consumo aparente del mercado norteamericano es abastecido por productos importados. La mayor parte de dichas importaciones proviene del mercado asiático, de países como China, Bangladesh y Vietnam, entre otros. (Ver cuadro 4)

Cuadro 4. Origen de las importaciones de Estados Unidos; variación 2006 - 2007 y participación 2007

\begin{tabular}{|c|c|c|c|}
\hline Source & Rank & $\begin{array}{l}\text { Change in } \\
\text { Import } \\
\text { Levels }\end{array}$ & $\begin{array}{l}\text { Share } \\
(2007)\end{array}$ \\
\hline World & & $3.5 \%$ & $100.0 \%$ \\
\hline China & 1 & $23.5 \%$ & $34.4 \%$ \\
\hline Bangladesh & 2 & $3.6 \%$ & $5.8 \%$ \\
\hline Vietnam & 3 & $34.5 \%$ & $5.5 \%$ \\
\hline Honduras & 4 & $7.6 \%$ & $5.2 \%$ \\
\hline Mexico & 5 & $-18.1 \%$ & $5.2 \%$ \\
\hline Indonesia & 6 & $5.0 \%$ & $4.6 \%$ \\
\hline India & 7 & $3.3 \%$ & $3.7 \%$ \\
\hline Cambodia & 8 & $2.8 \%$ & $3.7 \%$ \\
\hline El Salvador & 9 & $12.3 \%$ & $3.5 \%$ \\
\hline Pakistan & 10 & $3.4 \%$ & $3.0 \%$ \\
\hline Thailand & 11 & $-7.7 \%$ & $2.2 \%$ \\
\hline Philippines & 12 & $-22.3 \%$ & $2.0 \%$ \\
\hline Sri Lanka & 13 & $-9.2 \%$ & $1.8 \%$ \\
\hline Dom Republic & 14 & $-34.5 \%$ & $1.6 \%$ \\
\hline
\end{tabular}

Fuente: OTEXA. trade data imports and exports of textiles and apparel. http://otexa.ita.doc.gov/msrpoint.htm 


\section{Metodología}

Se aplicó una encuesta a 20 empresas exportadoras de confecciones a Estados Unidos. Las empresas se tomaron del registro de Proexport Colombia (consulta en línea realizada en el 2008) bajo los criterios de exportadores de confecciones de Santander a Estados Unidos; donde se encontró un total de 39 empresas exportadoras. Se incluyó dentro de las variables del proceso exportador las siguientes. (Ver cuadro $5)$.

Cuadro 5. Variables utilizadas en el estudio

\begin{tabular}{|c|c|c|c|}
\hline Variable & Definición & Indicador & Ítem \\
\hline \multirow{4}{*}{$\begin{array}{l}\text { Permanencia en el } \\
\text { mercado destino }\end{array}$} & \multirow{4}{*}{$\begin{array}{l}\text { Número de años que } \\
\text { lleva exportando }\end{array}$} & $0-1$ años & Muy Baja permanencia \\
\hline & & $(+) 1-3$ años & Baja Permanencia \\
\hline & & $(+) 3-5$ años & Permanencia media \\
\hline & & + de 5 años & Alta permanencia \\
\hline \multirow{2}{*}{$\begin{array}{l}\text { Forma de } \\
\text { exportación }\end{array}$} & \multirow{2}{*}{$\begin{array}{l}\text { Compromiso con el } \\
\text { proceso exportador }\end{array}$} & Directa & Directa (alto compromiso) \\
\hline & & Indirecta & Indirecta (bajo compromiso) \\
\hline \multirow{3}{*}{$\begin{array}{c}\text { Canal de } \\
\text { distribución }\end{array}$} & \multirow{3}{*}{$\begin{array}{l}\text { Mecanismo para hacer } \\
\text { llegar el producto al } \\
\text { consumidor final }\end{array}$} & Mayorista & Canal mayorista \\
\hline & & Minorista & Canal minorista \\
\hline & & Detallista & Canal detallista \\
\hline
\end{tabular}

Fuente. Elaboración propia

\section{Resultados}

Los resultados se presentan con base en la información obtenida en la aplicación de la encuesta a los empresarios exportadores de confecciones de Santander.

\section{Permanencia en el mercado de Estados Unidos}

De las empresas que actualmente realizan exportaciones al mercado norteamericano, el 90\% llevan más de cinco años en dicho mercado, lo que evidencia una alta tasa de permanencia. 10\% llevan entre 2 y 3 años en el mercado norteamericano. Como se ha dicho, una de las características del mercado es la alta exigencia del consumidor, por lo cual los productos que ingresan deben ser de muy alta calidad. Esto lleva a que la empresa que permanezca en el tiempo debe estar a la vanguardia en calidad, diseños y productividad, entre otros. 
Se ve, además, que no aparecen con frecuencia nuevas empresas exportadoras de confecciones al mercado norteamericano; lo que evidencia bajo compromiso de las empresas del sector para ingresar a este mercado. En algunas líneas de productos, se pueden aprovechar ventajas del mercado, sobre todo en aquellos renglones donde no hay tanta presencia de países asiáticos. La línea de producto predominante en las empresas exportadoras de Santander al mercado norteamericano, son los productos infantiles y para bebé, elaborados en tejidos plano y de punto.

Aunque la variable de medición de la permanencia exportadora, se tomó en años de exportación en el mercado norteamericano, se evidencia que las empresas exportadoras se han mantenido en el tiempo en dicho mercado; es decir, que las exportaciones no son esporádicas, más bien se sostienen en el tiempo.

Un hecho para aprovechar en el sentido industrial (conjunto de empresas productivas del sector), es la apropiación del conocimiento adquirido por parte de las empresas que han permanecido en dicho mercado, para que se conviertan en motor para impulsar nuevas exportaciones. Las experiencias individuales empresariales, deben convertirse en aprendizaje colectivo del sector confecciones para diseñar estrategias que ayuden a aumentar las exportaciones del sector.

\section{Aspectos de distribución física internacional}

El 95\% de los empresarios de confecciones de Santander a Estados Unidos, hace la exportación exclusivamente de manera directa, es decir, que no utiliza intermediarios como agencias o agentes en el proceso de exportación. El 5\% utiliza intermediarios. Es necesario aclarar que se utilizan servicios de intermediación para procesos logísticos (como transporte); y para servicios aduaneros (trámites). Al referirse al proceso de exportación directa, se entiende que los negocios y acuerdos son realizados entre la empresa y el comprador extranjero (en este caso el norteamericano), que pueden ser a través de contactos directos o por medio de vendedores o agentes en el extranjero.

El 50\% de los exportadores, ubica la mercancía en planta de origen para ser enviada a Estados Unidos, es decir, en el punto de fábrica del exportador. Otro 50\% ubica la mercancía en puerto de origen, en este caso es en el aeropuerto para ser enviado al mercado norteamericano.

\section{Conclusiones}

El mercado norteamericano es bastante atractivo para productos de la industria de confecciones debido al tamaño del mercado, a las exigencias del consumidor, al alto poder adquisitivo de la población, y al incremento de las importaciones de manera significativa. El tamaño del mercado norteamericano en confecciones 
alcanzó los 83.500 millones de dólares en el año 2008, con crecimiento del $30 \%$ aproximadamente entre el $2002-2008$.

Para el año 2008 Colombia exportó en confecciones a todo destino US\$1332 millones de dólares, y a Estados Unidos 285 millones que equivalen al 21,4\% de las exportaciones. Se aprecia que no existe una fuerte dependencia respecto al mercado norteamericano. Las exportaciones del sector confecciones de Colombia experimentaron un crecimiento de $133 \%$ entre los años 2002 - 2008; sin embargo, el desempeño de dichas exportaciones al mercado norteamericano aumentó solo $0,19 \%$. Es decir, que la industria de confecciones de Colombia aumentó en exportaciones a otros destinos, no así al mercado norteamericano. Esto, unido a la baja participación del sector en las importaciones de Estados Unidos, donde sólo se alcanzó el 0,43\% en el 2008, evidencia una baja competitividad del sector confecciones de Colombia en el mercado de Estados Unidos. En términos generales se aprecia que la industria de confecciones de Colombia y Santander, no aprovechan las ventajas derivadas del ATPDEA y más bien se pierde cada día participación y posicionamiento frente a otros países.

Las exportaciones de Santander de productos de confecciones a Estados Unidos alcanzaron en el año 2008 US\$ 7,7 millones de dólares aproximadamente, con participación del 2,7\% de las exportaciones del sector de Colombia a dicho mercado; cifra bastante insignificante frente a las oportunidades que ofrece el mercado y las ventajas de contar con preferencias arancelarias derivadas del ATPDEA. Aunque es muy baja la participación de Santander en las exportaciones a Estados Unidos en confecciones, hay empresas que han logrado posicionarse y permanecer en dicho mercado. Estas empresas se convierten, de manera espontánea en jalonadoras para que otras empresas se arriesguen a incursionar en el mercado norteamericano El análisis del proceso exportador de estas empresas santandereanas de la industria de confecciones a Estados Unidos refleja los siguientes aspectos:

- La permanencia en el mercado norteamericano de las empresas exportadoras en confecciones es alta; el 84\% lleva más de 5 años con exportaciones a ese país.

- El 95\% de los exportadores de confecciones hace las operaciones de comercio exterior de manera directa, es decir, sin la utilización de intermediarios.

- El canal de distribución mayorista (85\%) es el más utilizado por los exportadores de confecciones de Santander; además se exporta a minoristas $(10 \%)$ y a detallistas $(5 \%)$.

Lo anterior refleja que la mayoría de empresas santandereanas del sector confecciones se ubican en fase intermedia (de acuerdo al modelo de Uppsala) del proceso de internacionalización; caracterizada por exportar productos a mercados psicológicamente distantes y hacer las operaciones de comercio exterior de manera directa. 


\section{Referencias Bibliográficas}

Alonso J.A. y Donoso V. (1991). La competitividad de la empresa exportadora española. ICEX. Madrid.

Annual Statistical Analysis of the U.S. Apparel \& Footwear Industries (2008)

Daniels John y Radebaugh Lee (2000). Negocios internacionales. Octava edición. México. Pearson educación.

Galván S. I. (2003). La formación de la estrategia de selección de mercados exteriores en el proceso de internacionalización de las empresas. Tesis doctoral. Universidad de Las Palmas de Gran Canaria, España. Facultad de Ciencias Económicas y Empresariales

Rialp, Alex (1999). Los enfoques micro-organizativos de la internacionalización de la empresa: una revisión y síntesis de la literatura”. Información Comercial Española. No. 781, pp. 117-128.

Olivares M. A. (2005). La globalización y la internacionalización de la empresa: ¿Es necesario un nuevo paradigma? Estudios Gerenciales vol 21, No. 96. Icesi. Cali.

OTEXA. trade data imports and exports of textiles and apparel. http://otexa.ita.doc. gov/msrpoint.htm. Accesado en Septiembre 2009.

TradeStats Express: http://tse.export.gov Accesado entre febrero 2009 - octubre 2009

DIAN - Siex. (Sistema Estadístico de Comercio Exterior de Colombia). http:// websiex.dian.gov.co/ Accesado entre marzo 2009 - octubre 2009. 


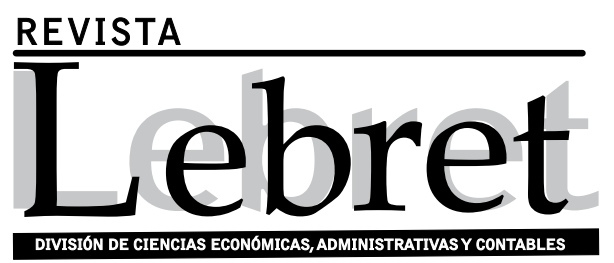

\section{CUPÓN DE SUSCRIPCIÓN}

\begin{tabular}{|l|}
\hline \multicolumn{1}{|c|}{ SUSCRIPTOR } \\
\hline Nombres \\
\hline Apellidos \\
\hline
\end{tabular}

\begin{tabular}{|c|c|c|}
\hline \multicolumn{3}{|c|}{ RESIDENCIA } \\
\hline \multicolumn{3}{|c|}{ Dirección } \\
\hline Ciudad & & País \\
\hline Telefono & fax & Celular \\
\hline
\end{tabular}

\begin{tabular}{|l|l|}
\hline & \multicolumn{2}{c|}{ SUSCRIPCIÓN ANUAL } \\
\hline Colombia $\$ 10.000$ & Exterior: US $\$ 5$ \\
\hline
\end{tabular}

\section{SUSCRIPCIÓN A PARTIR DEL NÚMERO}

\section{ESTIMADO SUSCRIPTOR}

- Consigne en la cuenta de ahorrros número 184-91-706-0 del Banco de Bogotá a nombre de Universidad Santo Tomás

- Envie copia del recibo de consignación, junto con este cupon a la División de Ciencias Económicas y Contables de la Universidad Santo Tomás en la carrera 18 № 9-27 Bucaramanga y copia a revistalebret@gmail.com 